\title{
SIGUE: Making Web Courses Adaptive
}

\author{
Cristina Carmona, David Bueno, Ricardo Conejo \\ Departamento de Lenguajes y Ciencias de la Computación \\ Universidad de Málaga \\ \{cristina, bueno, conejo\}@lcc.uma.es
}

\begin{abstract}
Most of the information of the WWW is not adaptive, rather it is dispersed and disorganized. Another difficulty is to find tools that help to create adaptive courses. SIGUE is an author tool that makes it possible to build adaptive courses using web pages that already exist. This means that if there is a lot of information on the web about the same topic the author doesn't have to design the content of a specific course, he can reuse these pages to build his own course, taking the best pages for the concepts he wants to explain. The author can also construct adaptive courses reusing previously non-adaptive ones. SIGUE provides an enhanced interface for the student, controls his interaction, and annotates the visited links in a student model.
\end{abstract}

\section{Introduction}

Nowadays, there are many tutorials on the WWW that let students acquire knowledge on a specific subject. Most of them are not adaptive. Because of the freedom and the disorganization of the $\mathrm{Web}$, a user can navigate freely in the pages of a course, and even in pages not related with the course. These problems have been stated several times and have been clearly summarized in [1], which points out four main problems: Disorientation, Cognitive overload, Narrative flow, and Content readiness. One of the characteristics that make a tutorial system adaptive is the possibility of modifying the sequencing or presentation of the course for each user according to their knowledge or preferences. Also the ability to recommend which unit they should do next. In this article we present SIGUE. This system is an author tool on the Web that can convert courses that weren't adaptive into adaptive ones. The original course could be a static one or can be created from existing pages from different sources. This lets authors make courses with the best information on the Web about a subject. SIGUE provides a student's interface that assists the students with adaptive tools when they navigate through the web pages. Another aspect of adaptivity is the multilingual interface of SIGUE, currently available in Spanish and English. SIGUE can also be integrated as a component of MEDEA [2]. MEDEA is an open system to develop Intelligent Tutorial Systems. It is composed of independent educative modules coordinated by a core that controls the instruction of the student. MEDEA can decide with is the best module to explain a concept in each moment. There are other modules, like SIETTE [3] which create tests to assess the estimated knowledge of the student. Besides SIGUE, there are also other tutorial systems on the Web that are adaptive and can guide the student 
through the learning process(ELM_ART [4], Hyperbook [5] or Metalinks[1]). The main difference between these systems and SIGUE is that SIGUE has not been developed as a content authoring tool with adaptive features, but as an authoring tool to reuse previously developed non-adaptive material, turning it into adaptive material.

\section{The SIGUE System}

SIGUE has two main modules, the author tool (SIGUEAUTOR) and the student tool (SIGUE), which is used by the student to study the course. Both of them are accessible through a WWW interface

\subsection{The author module (SIGUEAUTOR)}

The author module lets human tutors to create their Web courses through SIGUE. The domain model of the system and the way that users can create adaptive courses are explained in the following subsections.

Domain Model.- The author tool can create a course defining the domain model associated to it. For a specific course the domain model is a hierarchy of concepts. Various URLs can be associated with each concept and the relationship to other concepts can also be defined. For SIGUE two relationships have been defined: "belongs to" and "prerequisite of". This gives an idea of the order in which concepts should be visited, which is the sequence of the curriculum. The number of pages (URL) to describe a concept is unlimited. It is necessary to indicate the kind of information that each page contains (theory, examples or exercises) and the difficulty (easy, normal or hard).

Creation of Courses.- Once the domain model is created and all the concepts and relations have been defined, the author has to associate the pages for each concept. The authoring tool lets the user navigate freely, but with supervision. The pages visited by the user are processed by a parser that modifies the links in accordance with SIGUE. The parser modifies regular links and forms. When the author finally finds an appropriate page for a concept he only has to click a button to add it to the course. The author can also define a glossary of terms for the course with synonymous. When a term defined in the glossary appears in any page of the course, a link is automatically included to show the definition of this term. Courses in SIGUE can have different modes of operation. The author decides how adaptive the course will be and must set the mode of operation accordingly. There are four predefined modes: (a) Disable all the links in the pages shown to the student. He will be able to do the course by accessing documents only through the concept tree; (b) Leave all the links of the pages, this lets users navigate freely even in pages not related to the course; (c) Enable only the links that give references to pages that belongs to the course. (d) Full adaptive. The links will be enabled according to the user model. Only those links corresponding to concepts that the user is ready to learn will be activated. To construct an adaptive course, it is necessary to define the prerequisite relationship of the concepts. It is important to remark that the author of a course in SIGUEAUTOR doesn't necessarily need to be the author of the HTML pages that he associates to his 
courses. One of the main ideas of this system is to reuse and compile pages that are related to a topic, but are in different places on the WWW, logically, thereby creating an adaptive course with them.

\subsection{The student module (SIGUE)}

Once the course has been developed with SIGUEAUTOR, it will be available to students in SIGUE. While connected to the web through SIGUE, the student will see the hierarchical structure of concepts created by the author. Each time he selects a node he will see the main page associated with this concept. Before showing any page of the course, the HTML document is parsed and the links and submission forms are redirected to the SIGUE engine that acts as a supervisor between the user and the WWW. All links and forms are modified according to the strategy selected by the author for the course. The system will also include new references for the terms in the glossary, as defined by the author. If the course is fully adaptive, a student model is created for each user. In this model SIGUE stores the student's navigation path for each concept of the course. For each concept it shows two indicators. (a) The first one shows the estimated background of the student visiting this concept, that is, if he's prepared or not to read it, according to previous pages that have been visited. (b) The second is an indicator of the percentage of pages related to that concept that he has already visited. SIGUE makes estimations of the knowledge based on the percentage of visited pages for each concept. So, if the percentage of URLs visited for a concept is less than the minimum the status of the concept is "empty"; if this percentage of visited pages is bigger than the maximum, the status is "full". The intermediary case is shown as "half-full". The status of a concept is associated to the percentage of pages the user has studied within a concept. This information is shown to the user by a progress bar that appears next to each concept. The level of preparation necessary for a user to visit a concept is reflected by using colors in the nodes of the concept tree. They can be green, red or orange. The color of the node is decided using the status of the prerequisites in this way: Green: A concept has this status when all the prerequisites are shown to have their status as "full". The user is ready to learn it. Red: This will be the color if at least one of the prerequisites of a concept is "empty". It indicates that the user is not ready to study the concept. Orange: This indication will appear when no empty prerequisites exist. Nodes with this color could be studied but finishing all the prerequisites first is recommended. The student model is updated after each interaction, every time the user visits a page. This update is reflected in the concept tree, modifying the status and color of nodes accordingly. The aim of SIGUE is to guide the student's navigation, and support it with adaptive annotation, but at the same time let him move freely through the pages of the course. This is why access to any page is permitted for the user even if this is not recommended (red). When a concept is red, it won't change its color until its prerequisites have been visited, even if the user has already visited the documents for this concept. The student also has the freedom to visit pages through the concept tree or through the activated links of each page. The activated links correspond to the glossary and to the recommended concepts (green). This means that if there is a link in one page to a page of a concept that is not recommended (red, orange) the authors can decide whether this link should 
be activated or disabled. Another important characteristic used to guide students is the inclusion of a simple planner that the student can use to get the best recommendation for the next document to view from the concept he is studying. This mechanism is designed for "two buttons users". These are the NEXT and BACK buttons. An evaluation module can replace the simple mechanism used by SIGUE, when SIGUE is used as a component of the MEDEA architecture.In this case the navigation frame provided by SIGUE is replaced by MEDEA's navigation frame. The SIGUE component receives a temporary student model each time it is activated by a call from MEDEA, and this information is used to control the links on the main page.

\section{Conclusions and Future Works}

The web is a great source of information, but it is dispersed and hard to find. The advantage of SIGUE is the possibility of taking the best parts of each tutorial; compiling them in the order the author thinks is best; organizing them; adding relationships and glossary terms; and providing a learning environment in which the student is always oriented because someone (the course author) has followed the path before him and has provided some markers. The author can also decide the degree of freedom that the user should have while navigating, according to the characteristics of his expected audience. The adaptive features of SIGUE can also be used to convert static tutorials into adaptive tutorials. SIGUE is an independent and adaptive component, with author and student modules that can be integrated into MEDEA. If a better measure of the knowledge is needed it can be taken from MEDEA. At present the prototype of SIGUE is fully functional and can be used freely. Future work on SIGUE will be directed towards improving the estimation of user understanding by analyzing their navigation behavior. SIGUE could also a workbench to compare the results and the satisfaction of users studying a course in an adaptive way or not. It is available for trial at http://www.lcc.uma.es/SIGUE

\section{References}

1. Murray, T. Shen, T. Piemonte, J. Condit, C. Tibedau, J.: Adaptivity for conceptual and narrative flow in hyperbooks: The Metalink system. Adaptive Hypermedia and Adaptive Web-based system. Lecture Notes on Computer Science 1892, Berlin (2000) , 155-166.

2. Trella, M., Conejo, R., Bueno, D.: MEDEA: Una arquitectura basada en componentes para el desarrollo de Sistemas Tutores Inteligentes en Internet. CAEPIA 2001. (2001) 469-478.

3. A.Rios, E.Millán, M.Trella, J.Perez-de-la-Cruz, R.Conejo, Internet Based Evaluation System, en: Artificial Intelligence in Education, IOS Press, Amsterdam (1999) 387-394.

4. Weber, G., Specht, M.: User Modeling and Adaptive Navigation Support in WWW-Based Tutoring Systems. Proceedings of the Sixth International Conference on User Modeling, UM97. Springer Wien New York (1997) 289-300.

5. Henze, N. Nejdl, W.: Adaptivity in the KSB Hyperbook System. Proceedings of the $2^{\text {nd }}$ Workshop on Adaptive Systems and User Modeling on the WWW. (1999) 